\title{
Charge Carrier Transport and Generation via Trap-Mediated Optical Release in Organic Semiconductor Devices
}

\author{
Oskar J. Sandberg $\odot,{ }^{*}$ Stefan Zeiske, Nasim Zarrabi, Paul Meredith, and Ardalan Armin $\oplus^{\dagger}$ \\ Sustainable Advanced Materials (Sêr-SAM), Department of Physics, Swansea University, \\ Singleton Park, Swansea SA2 8PP Wales, United Kingdom
}

(Received 10 December 2019; revised manuscript received 13 February 2020; accepted 3 March 2020; published 25 March 2020)

\begin{abstract}
The impact of intermixed donor-acceptor domains in organic bulk heterojunction (BHJ) solar cells, using low-donor-content devices as model systems, is clarified. At low donor contents, the devices are found to exhibit anomalously high open-circuit voltages independent of the donor-acceptor energetics. These observations can be consistently explained by a theoretical model based on optical release of trapped holes, assuming the donors behave as trap sites in the gap of the acceptor. Our findings provide guidelines for reducing the large open-circuit voltage losses in organic solar cells and avoiding morphology-induced losses in state-of-the-art BHJ solar cells and photodetectors.
\end{abstract}

DOI: 10.1103/PhysRevLett.124.128001

Power conversion efficiencies exceeding $16 \%$ have recently been achieved for organic solar cells [1]. Upon photon absorption, strongly bound excitons with diffusion lengths of 5-10 $\mathrm{nm}$ are formed in the active absorber layer of these solar cells $[2,3]$. To convert excitons into free carriers, the most efficient cells are based on a concept unique to organic semiconductor devices: the bulk heterojunction (BHJ) in which the active absorber layer is composed of a phase-separated mixture of two organic semiconductors-an acceptor $(A)$ and a donor $(D)$, possessing an energy gradient at the $D-A$ interfaces. Once an exciton reaches a $D-A$ interface, this ultimately results in the generation of a free electron in the conduction level [referred to as lowest unoccupied molecular orbitals (LUMOs)] of the acceptor and a hole in the valence level [highest occupied molecular orbitals (HOMOs)] of the donor. However, $D-A$ systems suffer from the presence of additional intermediary charge-transfer (CT) states at the interfaces, which limit the open-circuit voltage $\left(V_{\text {oc }}\right)$ in these systems [3-5]. As these processes occur at $D-A$ interfaces, the morphology of the active layer is arguably the most important factor controlling device performance in organic solar cells $[6,7]$.

According to the general consensus, the donor-acceptor blend ideally forms percolating continuous donor and acceptor phases with $D-A$ phase separations of 5-10 nm $[2,3]$. This allows for the optimum number of excitons to be harvested at the $D-A$ interfaces, simultaneously providing pathways for the separated electrons and holes to be transported within the neat phases to the electrodes. In reality, however, the donor and acceptor phases form disordered networks with complicated morphologies, containing both neat and molecularly intermixed regions [8-11]. While some studies indicate that neat nanodomains of donor and acceptor are essential [12], other studies suggest that a certain degree of $D-A$ intermixing might be beneficial for charge generation facilitating the charge separation process at the $D-A$ interface [13-15]. However, if the length scale of the intermixed regions is much larger than the average charge hopping distance, free carriers are likely to become trapped within such regions, resulting in impaired charge transport and increased nongeminate recombination [16].

Recently, it was discovered that efficient charge collection and high $V_{\text {oc }}$ can also be realized in fullerene-based BHJ solar cells containing only trace amounts (1\%-10\% by molar content) of donor [17-19]. These so-called lowdonor-content solar cells consist of well-dispersed donor molecules embedded in a neat fullerene acceptor matrix, which is analogous to the intermixed regions of donor and acceptor molecules in BHJs, and as such, a useful model for studying phase mixing requirements. The mechanism of how holes, photogenerated in the donor, are transported to the electrode in these devices is currently under debate. It has been proposed that the hole transport is governed by thermal activation of holes from the donor to the acceptor [20], after which the photogenerated holes are transported in the acceptor HOMO [21]. Other studies have suggested that the hole transport remains dominated by direct donordonor hole transfer even for donor-donor site distances of $2-4 \mathrm{~nm}$, corresponding to $1.5 \%-10 \%$ donor molar contents [22-24].

However, relatively high external quantum efficiencies (EQEs) above $10 \%$ have still been observed at donor contents well below 1\% [25]. These same systems also show a nearly perfect quenching of photogenerated excitons for typical exciton diffusion lengths of 5-10 nm. Therefore, domains with ultralow donor contents still play a critical role in BHJ systems, as quenched excitons within these domains may represent a large fraction of the total 
photoexcitations. Whether or not the subsequent charge carriers, generated within these domains, can contribute to the photocurrent determines the overall performance of the BHJ system. In this Letter, we use low-donor-content devices as model systems to investigate the influence of $D-A$ intermixing in organic solar cells. At donor molar contents $\leq 1 \%$, an anomalously high $V_{\text {oc }}$, independent of the donor energetics, is observed. A theoretical model explaining these observations is developed, providing new insights into reducing both losses in the $V_{\text {oc }}$, as well as losses caused by morphology in organic solar cells, critical for the development of future donor-acceptor blends.

Figure 1 shows the experimental $V_{\text {oc }}$ at different donor content under AM1.5G incident illumination for organic solar cells based on the acceptor [6,6]-Phenyl $\mathrm{C}_{71}$ butyric acid methyl ester $\left(\mathrm{PC}_{71} \mathrm{BM}\right)$ and donors with different HOMO levels [4,4'-Cyclo hexylidenebis[N,N-bis(4-methylphenyl) benzenamine (TAPC), 9'-spirobi[9H-fluorene]2,2',7,7'-tetramine (Spiro-MeOTAD), and 4,4',4"-Tris[(3methylphenyl) phenylamino] triphenylamine (mMTDATA)], exhibiting different CT state energies $\left(E_{\mathrm{CT}}\right)$. The experimental details are given in the Supplemental Material [26]. For donor molar contents $\geq 5 \%$, the $V_{\text {oc }}$ depends strongly on the donor, conforming with the standard BHJ behavior where the $V_{\mathrm{oc}}$ is determined by the energetics at the $D-A$ interface $\left(E_{\mathrm{CT}}\right)$, $q V_{\mathrm{oc}} \approx E_{\mathrm{CT}}-0.6 \mathrm{eV}$, scaling linearly with the difference between the donor HOMO and acceptor LUMO $[4,5,32,33]$. This is consistent with the findings of a recent study on $\mathrm{C}_{60}$-based BHJs with similar donor content [27].

Figures 2(a) and 2(b) show the current-voltage $(J-V)$ characteristics under AM1.5G incident illumination and intensity-dependent normalized EQE, respectively, for devices with 5\% donor content. The EQEs are characterized by a continuous decrease with increasing irradiance $\left(I_{L}\right)$. As demonstrated in Fig. S5 [26], this behavior can be

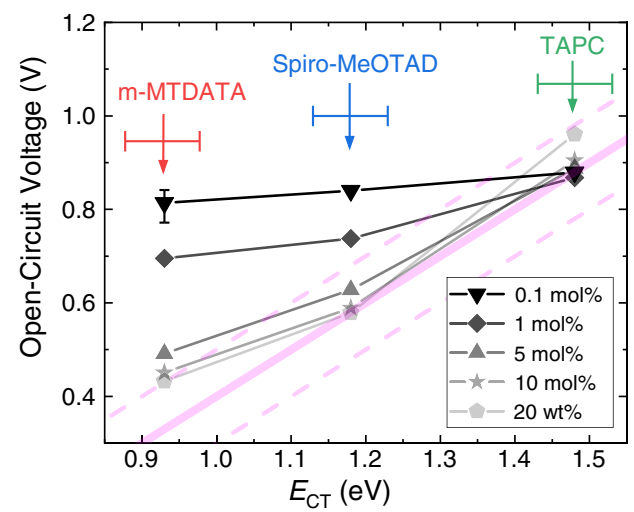

FIG. 1. Experimental $V_{\text {oc }}$ under one sun illumination versus the charge-transfer state energy $E_{\mathrm{CT}}$ of organic solar cells based on the acceptor $\mathrm{PC}_{71} \mathrm{BM}$ at varying content of donors with different HOMOs. The donor is either TAPC, Spiro-MeOTAD, or m-MTDATA. For comparison, $V_{\mathrm{oc}}=E_{\mathrm{CT}} / q-0.6 \mathrm{~V}( \pm 0.1 \mathrm{~V})$ is indicated by the pink solid line (dashed lines). reproduced with drift-diffusion simulations [30,31] assuming a standard BHJ, where electrons are transported in the acceptor and holes are transported through the donor, but with an effectively reduced hole mobility [26]. The degraded $\mathrm{EQE}$ at higher intensities is attributed to the low hole mobilities increasing the nongeminate recombination [28]. This hypothesis is supported by the m-MTDATA device showing an $\mathrm{EQE} \propto I_{L}^{-1 / 4}$ dependence, characteristic of space-charge-limited photocurrents caused by imbalanced mobilities [34] (note that a short-circuit current density $J_{\text {sc }} \propto I_{L}^{3 / 4}$ is expected in this case [35,36]). This agrees with the low hole mobility of $10^{-8} \mathrm{~cm}^{2} / \mathrm{V}$ s reported for m-MTDATA: $\mathrm{C}_{60}$ at similar donor contents [23].

While trapping-and-release transport of holes cannot be excluded for the TAPC case, owing to the shallow acceptordonor HOMO-HOMO offset (Supplemental Material, Table SI [26]), this process is expected to become increasingly less efficient for offsets larger than $0.3 \mathrm{eV}$, significantly increasing the likelihood of recombination [20]. Therefore, trapping and release of holes by thermal activation is unlikely in the Spiro-MeOTAD and m-MTDATA devices (exhibiting large HOMO-HOMO offsets) under realistic solar cell operating conditions, suggesting that the hole transport occurs by direct donor-donor charge transport at these donor contents [22], but with a reduced hole mobility [23].

To eliminate direct donor-donor hole transport, thus mimicking an ultralow domain impurity level in BHJ systems, we reduced the donor molar content down to $0.1 \%$, corresponding to a donor-donor separation of $10 \mathrm{~nm}$, which is beyond the range for direct donor-donor hole hopping. As evident from Fig. 1, upon decreasing the donor (a)

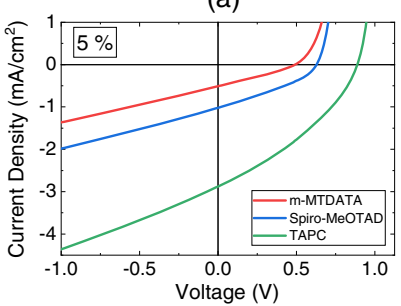

(c)

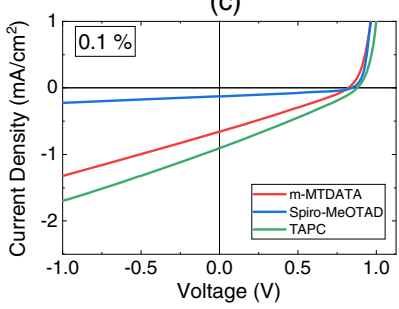

(b)

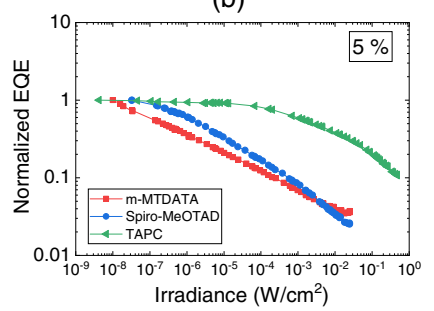

(d)

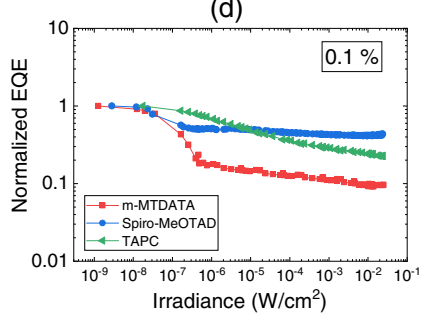

FIG. 2. Experimental $J-V$ characteristics under one sun illumination and light-intensity-dependent normalized EQEs of organic solar cells based on the acceptor $\mathrm{PC}_{71} \mathrm{BM}$ with $5 \%$ donor molar content are shown in (a) and (b), respectively. The corresponding cases for $0.1 \%$ donor molar contents are shown in (c) and (d). 
molar content from $5 \%$ to $0.1 \%$ a drastic increase of the $V_{\mathrm{oc}}$ is seen for the systems with large HOMO-HOMO offsets, the $V_{\mathrm{oc}}$ becoming independent of the $D$-A energetics $\left(E_{\mathrm{CT}}\right)$. This is most prominent for m-MTDATA where a $0.3-0.4 \mathrm{~V}$ increase is obtained, resulting in an open-circuit voltage of $V_{\text {oc }}=0.82 \mathrm{~V}$-anomalously large for this material system. Since the $D-A$ energetics of the blend remain relatively unchanged during this transition (Fig. S3 [26]), it becomes evident that this behavior cannot be described by the standard BHJ picture. Moreover, in contrast to the 5\% donor-content devices, the EQEs versus irradiance of the $0.1 \%$ donor-content devices show two approximately intensity-independent regions, as can be seen in Fig. 2(d) (cf. Supplemental Material [26] for additional experimental data).

To explain these observations, we take a closer look at the physical processes at play in the ultralow-donor-content limit, where nearest-neighbor donor site distances are large enough for direct donor-donor hole transfer to be negligible. The transport of free electrons and holes then exclusively takes place in the acceptor, whereas the donor sites act as traps for holes. A schematic energy level diagram is illustrated in Fig. 3(a). Here, $\Delta_{t}=E_{t}-E_{v}$ is the trap depth (HOMO-HOMO offset), $E_{g}^{D A}=E_{g}^{A A}-\Delta_{t}$ is the donor-acceptor energy gap, $E_{g}^{A A}=E_{c}-E_{v}$ is the acceptor-acceptor energy gap, and $E_{c}\left(E_{v}\right)$ is the LUMO (HOMO) level edge in the acceptor. A donor site is

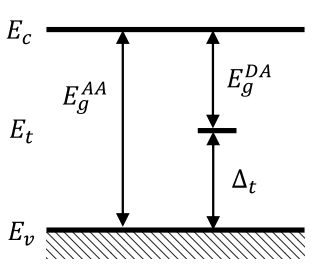

(a)

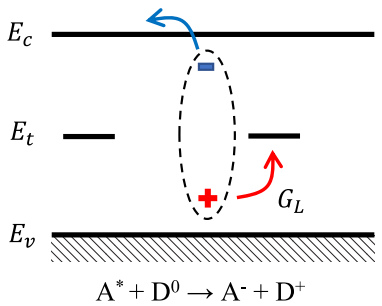

(c)

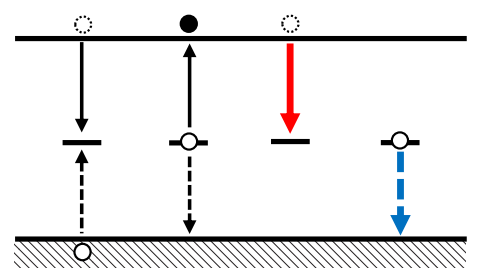

(b)

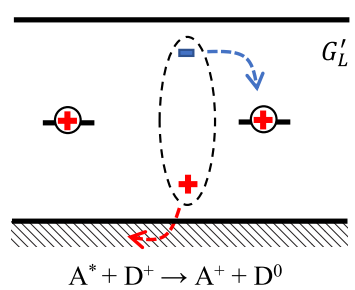

(d)
FIG. 3. (a) Schematic energy level diagram of an acceptor layer containing traplike donor sites. (b) The relevant kinetic processes for holes in the dark (black arrows) and under illumination; the arrows indicate the transitions of holes (electrons are simultaneously transferred in the opposite direction). The photogeneration of free electrons in the acceptor and holes in the donor is indicated by the red arrow, whereas the optical release of holes from donor to acceptor is indicated by the blue dashed arrow. (c),(d) The schematic picture of charge generation via traplike donor sites. positively charged when occupied by a hole and neutral when occupied by an electron (ground state). In the dark, under steady-state conditions, the hole occupation of the donor sites is described by Shockley-Read-Hall (SRH) statistics [37]; see Fig. 3(b). Accordingly, a trapped hole can either be released by thermal activation to the acceptor HOMO or recombine with a free electron from the acceptor LUMO. Similarly, a neutral donor site can either capture a free hole from the acceptor HOMO or obtain a hole directly from the acceptor LUMO as a result of thermal excitation of an electron (ground state) from the donor HOMO to the acceptor LUMO. Under illumination, the excitation rate of electrons from traplike donor sites is significantly enhanced due to the presence of photogenerated excitons.

Photogenerated excitons, induced by photons absorbed in the acceptor, are predominantly quenched and dissociate at donor sites, followed by a hole transfer to the donor. This results in the generation of a free electron in the acceptor and a (trapped) hole in the donor, with the rate $G_{L}$ [Fig. 3(c)]. However, this process requires that the donor site is in the ground state (neutral) prior to the charge transfer. If the donor site is initially occupied by a hole, we instead expect the charge-reversed process to occur; namely, a photoinduced electron transfer from the acceptor exciton to the positively charged donor site (now acting as an acceptor), which, after charge separation, produces a free hole in the acceptor HOMO and neutralizes the donor site. This optical release mechanism of trapped holes, depicted in Fig. 3(d), is assumed to occur with a rate $G_{L}^{\prime}$. To account for the donor site occupation of holes, we write

$$
\begin{aligned}
G_{L} & =G_{L, 0}\left[1-f_{t}\right], \\
G_{L}^{\prime} & =\gamma G_{L, 0} f_{t},
\end{aligned}
$$

where the prefactor $\gamma$ describes the relative strength of the CT state dissociation between the two processes (we assume $\gamma=1$ ), and $f_{t}$ is the fraction of donors occupied by a hole. Clearly, optical release is only relevant when a substantial fraction of donor sites is occupied by holes. In the limit when most donor sites are unoccupied $f_{t} \ll 1$ (assumed to be the case in standard BHJs), we expect $G_{L}=G_{L, 0}$ and $G_{L}^{\prime}=0$.

By accounting for these optical processes [38], the SRH kinetics can be modified and the net-generation-recombination rate of free electrons and holes in the acceptor, taking place via traplike donor sites, reads [26]

$$
\mathcal{U}_{D-A}=\frac{C_{p} C_{n} N_{t}}{C_{n}\left[n+n_{1}^{*}\right]+C_{p}\left[p+p_{1}^{*}\right]}\left[n_{1}^{*} p_{1}^{*}-n p\right],
$$

where

$$
\begin{aligned}
& n_{1}^{*}=N_{c} \exp \left(-\frac{E_{g}^{D A}}{k T}\right)+\frac{G_{L, 0}}{C_{n} N_{t}} ; \\
& p_{1}^{*}=N_{v} \exp \left(-\frac{\Delta_{t}}{k T}\right)+\frac{\gamma G_{L, 0}}{C_{p} N_{t}}
\end{aligned}
$$


Here, $C_{p}$ is the hole capture coefficient, $C_{n}$ is the freeelectron-trapped-hole recombination coefficient, and $N_{t}$ is the donor concentration (or trap density); $N_{c}$ and $N_{v}$ are the density of LUMO and HOMO states in the acceptor, respectively. Furthermore, based on Eq. (3), noting that the total generation is exactly balanced by recombination at open circuit $\left(\mathcal{U}_{D-A}=0\right)$, the following analytical approximation for $V_{\text {oc }}$ can be obtained [26]

$q V_{\mathrm{oc}}=E_{g}^{D A}-k T \ln \left\{\frac{C_{n} N_{t} N_{c}}{G_{L, 0}}\left[1+\exp \left(\frac{\Delta_{t}-\Delta_{t}^{*}}{k T}\right)\right]^{-1}\right\}$

where

$$
\Delta_{t}^{*}=-k T \ln \left(\frac{\gamma G_{L, 0}}{C_{p} N_{t} N_{v}}\right)
$$

may be interpreted as an optical demarcation depth [39].

To demonstrate the physical implications of the proposed model, we have implemented the trap-assisted generation mechanism into the drift-diffusion model, assuming the generation of free charge carriers in the acceptor to exclusively take place via this mechanism (the excitons are quenched at donor sites); the details of the simulations are given in the Supplemental Material [26]. In Fig. 4(a), the $J-V$ curves of an organic solar cell based on an acceptor layer with traplike donors is simulated at different $\Delta_{t}$. The corresponding $V_{\mathrm{oc}}$, simulated in Fig. 4(b), agrees well with the analytical prediction Eq. (5). The intensitydependent normalized EQE (at short circuit) is simulated in the inset of Fig. 4(b). Overall, the observed experimental features (Figs. 1 and 2) at the lowest donor contents are well reproduced by the theoretical model (Fig. 4), supporting the (a)

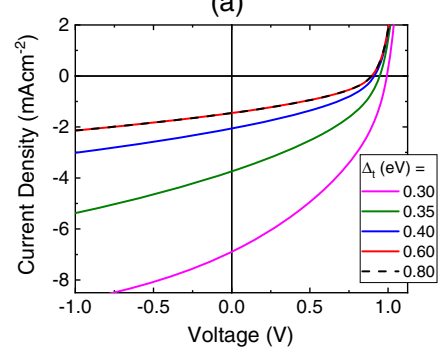

(b)

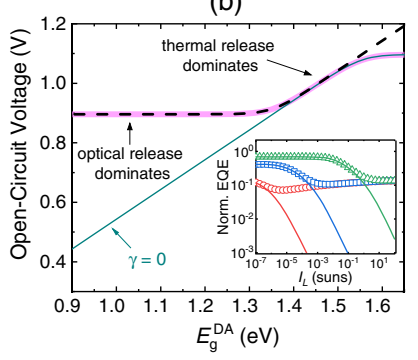

FIG. 4. (a) Simulated $J-V$ characteristics of an acceptor-based organic solar cell with traplike donors at different $\Delta_{t}$, assuming $N_{t}=10^{18} \mathrm{~cm}^{-3}$. (b) The corresponding simulated $V_{\text {oc }}$ versus $E_{g}^{D A}=E_{g}^{A A}-\Delta_{t}$ (bold solid line), alongside Eq. (5) (dashed line), for $E_{g}^{A A}=1.75 \mathrm{eV}$. (Inset) Simulated intensity-dependent EQEs (at short circuit), normalized to the case when all generated electron-hole pairs are extracted, for $\Delta_{t}=0.4 \mathrm{eV}$ (green triangles), $\Delta_{t}=0.6 \mathrm{eV}$ (blue squares), and $\Delta_{t}=0.75 \mathrm{eV}$ (red circles). For comparison, the cases without optical release $(\gamma=0)$ are indicated by the corresponding solid lines. presence of optical release via donors, acting as traps, in these systems. We note that the qualitative behavior in Fig. 4 is insensitive to injection barriers at the anode contact (see Fig. S8 [26]).

A closer inspection reveals that depending on the interplay between $\Delta_{t}^{*}$ and the trap depth $\Delta_{t}$ two distinctly different regimes can be identified. At low intensities or shallow HOMO-HOMO offsets, when $\Delta_{t}^{*} \gg \Delta_{t}$, corresponding to $f_{t} \ll 1$, the hole transport is governed by trapping and release by thermal activation. In this limit, holes in the donor and the acceptor are in equilibrium with each other and described by a common quasi-Fermi level, while the generation of free carriers is dominated by the "conventional" process depicted in Fig. 3(c). Accordingly, the $V_{\mathrm{oc}}$ [Eq. (5)] is identical to the one expected for standard BHJs with $q V_{\text {oc }} \propto E_{g}^{D A}$. Furthermore, the shape of the $J-V$ curve, the charge collection efficiency (normalized EQE), and the $V_{\text {oc }}$ are all dependent on $\Delta_{t}$. This type of behavior is expected to prevail as long as optical release remains negligible [or is neglected, $\gamma=0$; see Fig. 4(b)], consistent with previous analyses where optical release was not considered [20].

At higher intensities and/or larger HOMO-HOMO offsets when $\Delta_{t}^{*}<\Delta_{t}$, a significant fraction of donor sites are occupied by positively charged holes [see Fig. 3(d)], and optical release starts to dominate the detrapping of holes. Since beyond this point trapped holes are governed by an effective trap depth $\Delta_{t}^{*}$, the charge transport becomes independent of the actual trap depth. This explains the appearance of the second plateau in the normalized EQE, observed both experimentally and numerically, at higher intensities. In this regime $\left(\Delta_{t}^{*} \ll \Delta_{t}\right)$, two excitons are, on average, required to generate a free electron-hole pair (in the acceptor). While the first exciton produces a free electron in the acceptor and a trapped hole in the donor [Fig. 3(c)], the second exciton is needed to promote the trapped hole to the acceptor HOMO [Fig. 3(d)], manifested as an effective halving of the generation rate of free carriers in the acceptor (assuming $\gamma=1$ ).

Furthermore, $V_{\text {oc }}$ becomes independent of the energetics of the traplike donors when $\Delta_{t}^{*} \ll \Delta_{t}: q V_{\text {oc }} \propto E_{g}^{A A}$ as per Eq. (5). Instead, the $V_{\mathrm{oc}}$ is solely governed by the energetics of the acceptor in this limit, manifested as an increased $V_{\text {oc }}$ at ultralow donor contents, consistent with what is observed in Fig. 1. In this case, (trapped) holes in the donor are no longer in thermal equilibrium with (free) holes in the acceptor and thus described by separate quasi-Fermi levels [40]. This ultimately decouples the $V_{\text {oc }}$ from its dependence on the donor-acceptor CT energetics and associated losses; this is clearly observed for the m-MTDATA case (as detailed in Table SII [26]), corroborating the presence of optical release in these devices [see also Fig. S1(b) [26] ]. Optical release may thus present a key to overcome the large losses caused by nonradiative CT state recombination limiting $V_{\text {oc }}$ in organic solar cells [5,32]. 
Finally, apart from the high $V_{\text {oc }}$ typically observed in low-donor-content BHJs [17-19,41], independent of the donor HOMO, optical release of holes also explains how holes are able to be collected in devices or domains with ultralow donor concentrations. We emphasize that this type of optical release is expected to occur whenever a significant fraction of (donor) sites is occupied (by holes), e.g., at high enough intensities. In fact, this process is highly relevant for any situation where charges are energetically or spatially trapped within a BHJ, including carriers trapped in isolated donor (or acceptor) molecules or islands within neat domains, intermixed regions, or extrinsic trap states. These are likely present in all BHJs [12,42-44], being of particular importance in nonfullerene systems based on highly miscible components. Furthermore, as these "impurities" also act as exciton quenching centers, a considerable amount of excitons may be quenched at these sites [25]. It should be stressed that optical release still presents a loss mechanism for excitons. In order to avoid these morphology-induced losses in organic solar cells, it is therefore crucial to minimize the acceptor-donor HOMO-HOMO offset and, interestingly, this is what is empirically happening in the field of nonfullerene acceptor solar cells [45].

In conclusion, we have used low-donor-content BHJ solar cells as model systems to investigate the impact of intermixed domains in organic solar cells. For donor molar contents below $1 \%$, the donors increasingly behave as trap sites in the gap of the acceptor. To explain the device physics in this limit, a theoretical model based on optical release of trapped holes is proposed. Based on this model, we derive analytical expressions explaining the high $V_{\text {oc }}$ encountered in these devices. These findings not only improve our understanding of the effect of morphology, being of paramount importance in state-of-the-art BHJ solar cells and photodetectors, but also provide tools for reducing the large open-circuit voltage losses in organic solar cells.

This work was supported by the Sêr Cymru Program through the European Regional Development Fund, Welsh European Funding Office, and Swansea University strategic initiative in Sustainable Advanced Materials. A. A. received support from a Sêr Cymru II Rising Star Fellowship and P.M. is a Sêr Cymru II National Research Chair.

*o.j.sandberg@swansea.ac.uk

†ardalan.armin@swansea.ac.uk

[1] M. A. Green, E. D. Dunlop, D. H. Levi, J. Hohl-Ebinger, M. Yoshita, and A. W. Y. Ho-Baillie, Prog. Photovoltaics 27, 565 (2019).

[2] C. Deibel and V. Dyakonov, Rep. Prog. Phys. 73, 096401 (2010).

[3] L. Dou, J. You, Z. Hong, Z. Xu, G. Li, R. A. Street, and Y. Yang, Adv. Mater. 25, 6642 (2013).
[4] K. Vandewal, K. Tvingstedt, A. Gadisa, O. Inganäs, and J. V. Manca, Phys. Rev. B 81, 125204 (2010).

[5] J. Benduhn, K. Tvingstedt, F. Piersimoni et al., Nat. Energy 2, 17053 (2017).

[6] Y. Liu, J. Zhao, Z. Li, C. Mu, W. Ma, H. Hu, K. Jiang, H. Lin, H. Ade, and H. Yan, Nat. Commun. 5, 5293 (2014).

[7] K. Jiang, Q. Wei, J. Y. L. Lai, Z. Peng, H. K. Kim, J. Yuan, L. Ye, H. Ade, Y. Zou, and H. Yan, Joule 3, P3020 (2019).

[8] C. Müller, J. Bergqvist, K. Vandewal et al., J. Mater. Chem. 21, 10676 (2011).

[9] J. Zhao, A. Swinnen, G. Van Assche, J. Manca, D. Vanderzande, and B. V. Mele, J. Phys. Chem. B 113, 1587 (2009).

[10] W. Ma, J. R. Tumbleston, M. Wang, E. Gann, F. Huang, and H. Ade, Adv. Energy Mater. 3, 864 (2013).

[11] B. A. Collins, Z. Li, J. R. Tumbleston, E. Gann, C. R. McNeill, and H. Ade, Adv. Energy Mater. 3, 65 (2013).

[12] J. Zhang, H. Shuan Tan, X. Guo, A. Facchetti, and H. Yan, Nat. Energy 3, 720 (2018).

[13] J. K. Gallaher, S. K. K. Prasad, M. A. Uddin, T. Kim, J. Y. Kim, H. Y. Woo, and J. M. Hodgkiss, Energy Environ. Sci. 8, 2713 (2015).

[14] A. C. Jakowetz, M. L. Böhm, A. Sadhanala, S. Huettner, A. Rao, and R. H. Friend, Nat. Mater. 16, 551 (2017).

[15] S. Sweetnam, K. R. Graham, G. O. Ngongang Ndjawa, T. Heumüller, J. A. Bartelt, T. M. Burke, W. Li, W. You, A. Amassian, and M. D. McGehee, J. Am. Chem. Soc. 136, 14078 (2014).

[16] J. A. Bartelt, Z. M. Beiley, E. T. Hoke et al., Adv. Energy Mater. 3, 364 (2013).

[17] M. Zhang, H. Wang, H. Tian, Y. Geng, and C. W. Tang, Adv. Mater. 23, 4960 (2011).

[18] B. Yang, F. Guo, Y. Yuan, Z. Xiao, Y. Lu, Q. Dong, and J. Huang, Adv. Mater. 25, 572 (2013).

[19] K. Vandewal, J. Widmer, T. Heumueller, C. J. Brabec, M. D. McGehee, K. Leo, M. Riede, and A. Salleo, Adv. Mater. 26, 3839 (2014).

[20] T. Albes, L. Xu, J. Wang, J. W. P. Hsu, and A. Gagliardi, J. Phys. Chem. C 122, 15140 (2018).

[21] W. Feng, C. Song, X. Hu, S. Liu, R. Yi, X. Yang, H. Yan, and X. Hou, ACS Appl. Mater. Interfaces 10, 28256 (2018).

[22] A. Melianas, V. Pranculis, D. Spoltore, J. Benduhn, O. Inganäs, V. Gulbinas, K. Vandewal, and M. Kemerink, Adv. Energy Mater. 7, 1700888 (2017).

[23] D. Spoltore, A. Hofacker, J. Benduhn et al., J. Phys. Chem. Lett. 9, 5496 (2018).

[24] K. Ding, X. Liu, and S. R. Forrest, Nano Lett. 18, 3180 (2018).

[25] N. Zarrabi, A. Yazmaciyan, P. Meredith, I. Kassal, and A. Armin, J. Phys. Chem. Lett. 9, 6144 (2018).

[26] See Supplemental Material at http://link.aps.org/ supplemental/10.1103/PhysRevLett.124.128001 for experimental details, additional measurements and simulations, and analytical derivations and approximations, which includes Refs. [27-32].

[27] E. Collado-Fregoso, S. N. Pugliese, M. Wojcik et al., J. Am. Chem. Soc. 141, 2329 (2019).

[28] M. Stolterfoht, A. Armin, B. Philippa, and D. Neher, J. Phys. Chem. Lett. 7, 4716 (2016). 
[29] S. Zeiske, C. Kaiser, P. Meredith, and A. Armin, ACS Photonics 7, 256 (2020).

[30] O. J. Sandberg, M. Nyman, and R. Österbacka, Phys. Rev. Applied 1, 024003 (2014).

[31] O. J. Sandberg, A. Sundqvist, M. Nyman, and R. Österbacka, Phys. Rev. Applied 5, 044005 (2016).

[32] M. Azzouzi, T. Kirchartz, and J. Nelson, Trends Chem. 1, 49 (2019).

[33] J. Widmer, M. Tietze, K. Leo, and M. Riede, Adv. Funct. Mater. 23, 5814 (2013).

[34] The EQE is related to the photocurrent $\left(J_{\mathrm{ph}}\right)$ via $\mathrm{EQE}=\left(J_{\mathrm{ph}} / I_{L}\right) \times\left(E_{\mathrm{ph}} / q\right)$, where $E_{\mathrm{ph}}$ is the photon energy. For mobility-induced space-charge-limited photocurrents, having $J_{\mathrm{ph}} \propto I_{L}^{3 / 4}$ (see Refs. [35,36]), we therefore expect $\mathrm{EQE} \propto I_{L}^{-1 / 4}$.

[35] A. M. Goodman and A. Rose, J. Appl. Phys. 42, 2823 (1971).

[36] V. D. Mihailetchi, J. Wildeman, and P. W. M. Blom, Phys. Rev. Lett. 94, 126602 (2005).
[37] P. Würfel, Physics of Solar Cells (Wiley-VCH, Weinheim, Germany, 2009).

[38] Y. K. Hsieh and H. C. Card, J. Appl. Phys. 65, 2409 (1989).

[39] M. A. Lampert and P. Mark, Current Injection in Solids (Academic Press, New York, 1970).

[40] W. Shockley and W. T. Read, Phys. Rev. 87, 835 (1952).

[41] S. Sutty, G. Williams, and H. Aziz, J. Photonics Energy 4, 040999 (2014).

[42] C. Müller, T. A. M. Ferenczi, M. Campoy-Quiles, J. M. Frost, D. D. C. Bradley, P. Smith, N. Stingelin-Stutzmann, and J. Nelson, Adv. Mater. 20, 3510 (2008).

[43] B. A. Collins, J. R. Tumbleston, and H. Ade, J. Phys. Chem. Lett. 2, 3135 (2011).

[44] L. Ye, H. Hu, M. Ghasemi, T. Wang et al., Nat. Mater. 17, 253 (2018).

[45] R. S. Gurney, D. G. Lidzey, and T. Wang, Rep. Prog. Phys. 82, 036601 (2019). 